Additional material is published online only. To view please visit the journal online (http://dx.doi.org/10.1136/ gutjnl-2016-311585).

For numbered affiliations see end of article.

\section{Correspondence to} Dr Gregory B Lesinski, Department of Hematology and Medical Oncology, Winship Cancer Institute of Emory University, Building C, Room C3090, 1365 Clifton Rd. NE, Atlanta GA 30322, USA gregory.b.lesinski@emory.edu

Received 02 February 2016 Revised 13 September 2016 Accepted 03 October 2016 Published Online First 21 October 2016

\title{
IL-6 and PD-L1 antibody blockade combination therapy reduces tumour progression in murine models of pancreatic cancer
}

Thomas A Mace, ${ }^{1}$ Reena Shakya, ${ }^{2}$ Jason R Pitarresi, ${ }^{3}$ Benjamin Swanson, ${ }^{4}$ Christopher W McQuinn, ${ }^{1}$ Shannon Loftus, ${ }^{1}$ Emily Nordquist, ${ }^{1}$ Zobeida Cruz-Monserrate, ${ }^{1}$ Lianbo Yu, ${ }^{5}$ Gregory Young, ${ }^{6}$ Xiaoling Zhong, ${ }^{7}$ Teresa A Zimmers, ${ }^{7}$ Michael C Ostrowski, ${ }^{3}$ Thomas Ludwig, ${ }^{3}$ Mark Bloomston, ${ }^{8}$ Tanios Bekaii-Saab, ${ }^{9}$ Gregory B Lesinski ${ }^{10}$

\section{ABSTRACT}

Objective Limited efficacy of immune checkpoint inhibitors in pancreatic ductal adenocarcinoma (PDAC) has prompted investigation into combination therapy. We hypothesised that interleukin 6 (IL-6) blockade would modulate immunological features of PDAC and enhance the efficacy of anti-programmed death-1-ligand 1 (PD-

L1) checkpoint inhibitor therapy.

Design Transcription profiles and IL-6 secretion from primary patient-derived pancreatic stellate cells (PSCs) were analyzed via Nanostring and immunohistochemistry, respectively. In vivo efficacy and mechanistic studies were conducted with antibodies (Abs) targeting IL-6, PD-L1, CD4 or CD8 in subcutaneous or orthotopic models using Panc02, MT5 or KPC-luc cell lines; and the aggressive,

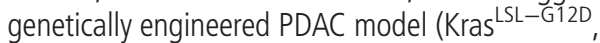
Trp53 ${ }^{\mathrm{LS} L-\mathrm{R} 270 \mathrm{H}}$, Pdx1-Cre, Brca2 ${ }^{\mathrm{F} / \mathrm{F}}$ (KPC-Brca2 mice)). Systemic and local changes in immunophenotype were measured by flow cytometry or immunohistochemical analysis.

Results PSCs ( $n=12)$ demonstrated prominent IL-6 expression, which was localised to stroma of tumours. Combined IL-6 and PD-L1 blockade elicited efficacy in mice bearing subcutaneous MT5 $(p<0.02)$ and Panc02 tumours ( $p=0.046)$, which was accompanied by increased intratumoural effector $T$ lymphocytes (CD62 $\mathrm{L}^{-} \mathrm{CD}_{4}{ }^{-}$). CD8-depleting but not CD4-depleting Abs abrogated the efficacy of combined IL- 6 and PD-L1 blockade in mice bearing Panc02 tumours $(p=0.0016)$. This treatment combination also elicited significant antitumour activity in mice bearing orthotopic KPC-luc tumours and limited tumour progression in KPC-Brca2 mice $(p<0.001)$. Histological analysis revealed increased T-cell infiltration and reduced $\alpha$-smooth muscle actin cells in tumours from multiple models. Finally, IL-6 and PD-L1 blockade increased overall survival in KPC-Brca2 mice compared with isotype controls ( $p=0.0012)$.

Conclusions These preclinical results indicate that targeted inhibition of IL-6 may enhance the efficacy of anti-PD-L1 in PDAC.

\section{INTRODUCTION}

Pancreatic ductal adenocarcinoma (PDAC) is expected to surpass breast and colon cancer,

\section{Significance of this study}

What is already known on this subject?

- Checkpoint blockade immunotherapy has limited efficacy in patients with pancreatic cancer.

- The proinflammatory cytokine interleukin 6 (IL-6) is highly elevated in patients with pancreatic cancer and is a prognostic factor for outcome.

\section{What are the new findings?}

- Pancreatic stellate cells are a major source of IL-6 in the pancreatic tumour microenvironment.

- Blockade of IL-6 and programmed death-1-ligand 1 (PD-L1) limits tumour progression and enhances overall survival in aggressive murine models of pancreatic cancer.

- Combination therapy increased circulating cells with Th1 phenotypic characteristics and intratumoural effector T cells.

- Combined blockade of IL-6 and PD-L1 immunotherapy elicits efficacy in a $\mathrm{CD} 8^{+}$ T-cell-dependent manner.

How might it impact on clinical practice in the foreseeable future?

- These preclinical results elucidate a novel combination therapy that could be translated to the clinic.

- Our results suggest that targeting IL-6 could improve responses of patient with pancreatic cancer to checkpoint blockade immunotherapy.

becoming the second leading cause of cancerrelated deaths by the year $2030 .{ }^{1}$ One of the major difficulties with PDAC is its clinical silence. Typically, the disease only becomes apparent after the tumour invades surrounding tissues or metastasises to distant organs. ${ }^{2}$ For many years, the current standard of care for most advanced patients with PDAC has been gemcitabine-based regimens including combined therapy with nab-paclitaxel 
(Abraxane) $^{3}$ or aggressive chemotherapy (eg, FOLFIRINOX) as a strategy to debulk the tumour and improve candidacy for surgery. Regardless, these advances may rightfully be classified as only incremental and justify further research to identify novel strategies with potential for long-term clinical responses and cures for this devastating malignancy.

Signalling downstream of interleukin 6 (IL-6) is important in PDAC genesis and progression. ${ }^{4} 5$ This pleiotropic cytokine binds membrane receptor complexes containing the common signal transducing receptor chain GP130 (glycoprotein 130), ${ }^{6}$ thereby initiating a complex series of signalling events that include the JAK/STAT, MAPK and phosphoinositide 3-kinase (PI3K) pathways. ${ }^{78}$ In particular, STAT3 is activated via phosphorylation at $\mathrm{Tyr}^{705}$ in most human PDAC specimens and cooperates with activated Kras to drive initiation and progression of PDAC in murine models. ${ }^{9} 10$ The IL-6/STAT3 axis can simultaneously promote the expansion of immunosuppressive cells or alter the balance of T-cell subsets. Among the most notable of these subsets are myeloid-derived suppressor cells (MDSCs) and T regulatory cells, given their prominent expansion and role as poor prognostic indicators in patients with advanced GI cancer. ${ }^{11-13}$ Interestingly, data from our group and others point to the pancreatic stroma as one likely source of IL-6. This cytokine is produced in abundance by components of the stroma including pancreatic stellate cells (PSCs) and tumourassociated myeloid cells. ${ }^{5}{ }^{14}$ In this manner, IL-6 can cooperate with other cytokines, either systemically or in the tumour microenvironment, to further amplify immune changes in patients. Recent studies using an inducible Kras-mediated PDAC murine model also showed that IL-6 was instrumental for PDAC progression. ${ }^{4}{ }^{15}$ In fact, lack of IL- 6 completely ablated cancer progression, even in the presence of oncogenic Kras. ${ }^{4}$ In agreement with these data, recent results from our group emphasise the importance of systemic IL- 6 in patients with PDAC. ${ }^{16}$ We analysed plasma from untreated patients with metastatic or nonresectable PDAC and found that IL-6, IL-10 and MCP-1 were significantly associated with overall survival, with IL-6 having a strong inverse relationship. ${ }^{16}$

There remains optimism that the dramatic efficacy of immunotherapy in other cancers may be realised in patients with PDAC. Indeed, blockade of T-cell checkpoint receptors with neutralising antibodies (Abs) has emerged as a promising immunotherapeutic approach. ${ }^{17}{ }^{18}$ Programmed death-1-ligand 1 (PD-L1), also known as B7-H1, is a cell surface protein and one of two ligands for programmed death receptor 1 (PD-1), a costimulatory molecule that negatively regulates T-cell responses. $^{19} 20$ Ligation of PD-L1 on cancer cells to PD-1 expressed on T cells suppresses T-cell activation and proliferation, and can induce apoptosis. Intratumoural expression of PD-L1 correlates with poor prognosis in patients with PDAC. ${ }^{17} 21$ Indeed, increased PD-L1 expression by cancer cells or the stroma is a fundamental escape mechanism from host immunity.

In theory, IL-6 blockade may modulate immunological features of PDAC in both the systemic circulation and the tumour microenvironment, thereby enhancing antitumour activity of anti-PD-L1. IL-6 blocking $\mathrm{Ab}$ is an attractive component of combination therapy regimens for PDAC for a number of reasons. First, IL-6 targeted Abs are FDA approved for other indications and could easily be repurposed for oncology. Second, data from our group and others suggest that IL-6 is a major factor produced by multiple cellular compartments to promote immune suppression and PDAC progression. ${ }^{17} 22-24$ Finally, blocking IL- 6 may tune the phenotypic properties of both myeloid and T-cell compartments of a tumour-bearing host to be more amenable to efficacy from immunotherapy regimens.

This preclinical study tested the hypothesis that IL-6 blockade would enhance the efficacy of anti-PD-L1 checkpoint inhibitor therapy in preclinical models of PDAC. A significant benefit was observed from this therapy in the subcutaneous MT5 and Panc02 models, an orthotopic KPC luciferase model and an aggressive genetically engineered mouse model (GEMM) of PDAC. Mechanistic studies revealed increased pancreatic effector T-cell infiltration and efficacy dependent on $\mathrm{CD} 8^{+} \mathrm{T}$ cells. These results indicate that targeting IL- 6 may enhance the efficacy of Abs targeting PD-L1 in PDAC by improving the phenotypic properties and infiltration of T cells.

\section{EXPERIMENTAL METHODS}

\section{Cell lines and reagents}

The murine pancreatic cancer cell line Panc02 was purchased from the ATCC and cultured in RPMI (Gibco) with 10\% fetal bovine serum (FBS), $10 \mathrm{mM}$ l-glutamine and antibiotics. Murine MT5 (Kras ${ }^{\text {LSL-G12D }}$, Trp53 $3^{\text {LSL-R270H }}$ and Pdx1-cre) pancreatic cells were a kind gift from Dr. Tuveson (Cold Spring Harbor Laboratory, Cold Spring Harbor, New York, USA) and cultured in RPMI (Gibco) with 10\% FBS, $10 \mathrm{mM}$ l-glutamine and antibiotics. KPC-luc cells for orthotopic experiments were derived from KPC mice (Kras ${ }^{\mathrm{LSL}-\mathrm{G} 12 \mathrm{D}}$, Trp $53^{-/-}$and PDX-1-Cre) and transfected with enhanced firefly luciferase as previously described. $^{25}$ Murine isotype controls (clone 3E5.2H12), anti-IL-6R (clone BP-5875) and anti-PD-L1-blocking Abs were obtained from either Genentech (San Francisco, California, USA) for in vivo studies in the KPC-Brca2 murine model. Murine Abs to IL-6 (clone MP5-20F3), PD-L1 (clone 10F.9G2) or isotype controls (clones LTF-2 and HRPN) were purchased from BioXcell (West Lebanon, New Hampshire, USA) for in vivo studies using the MT5, Panc02 and KPC-luc cell lines.

\section{Murine models of pancreatic cancer}

KPC-Brca2 mice were generated by interbreeding $\mathrm{BrCa}^{\text {flox2/flox2 }}$; $\mathrm{Kras}^{L S L-G 12 D /+}$ with Brca2 $2^{\text {flox2/flox2 }}$, Trp $53^{L S L-R 270 H /+}$ and Pdx1-cre animals. $^{26}$ The mouse strains $p 53^{L S L-R 270 H}$ (strain number 01XM3), Kras ${ }^{L S L-G 12 D}$ (strain number 01XJ6) and Pdx1-cre (strain number 01XL5) were acquired from the National Cancer Institute Frederick Mouse Repository. All transgenic mice generated in this study were maintained on a mixed 129/B6 genetic background. All studies involving MT5, Panc02 and KPC-luc tumours used syngeneic, female C57BL/6 mice, 5-6 weeks of age.

\section{In vivo efficacy studies}

KPC-Brca2 mice (5 weeks of age) were treated with isotype controls, anti-IL-6R and/or anti-PD-L1 Abs (Genentech) at a dose of $200 \mu \mathrm{g} /$ mouse, three times each week (Monday, Wednesday and Friday). Following 2 weeks of treatment, animals were euthanised via $\mathrm{CO}_{2}$ asphyxiation, followed by cardiac puncture. Plasma, splenocytes and tumour tissue were collected for further analysis. Pathology was assessed in H\&E-stained slides to determine the differentiation state of tissue as pancreatic intraepithelial neoplasia (PanIN) 1A, PanIN 1B, PanIN 2 or PDAC. For studies using MT5 and Panc02 tumours, $1 \times 10^{6}$ or $3 \times 10^{5}$ cells, respectively, were injected subcutaneously in the flank of C57BL/ 6 mice three times each week with $200 \mu \mathrm{g} /$ mouse of isotype, anti-IL-6 or anti-PD-L1 Abs (BioXCell) Ab treatment starting once tumours reached $50-100 \mathrm{~mm}^{3}$ volume. For orthotopic studies, C57BL/ 6 mice were injected with $1 \times 10^{6} \mathrm{KPC}-$ luc (luciferase expressing) cells in Matrigel (BD Biosciences) in the tail of the pancreas. Tumour growth was analysed once in a week by 
bioluminescent imaging and end of study tumour weight was determined immediately postmortem. Mice were treated three times each week with $200 \mu \mathrm{g} /$ mouse of isotype, anti-IL-6 or anti-PD-L1 Abs (BioXCell). For T-cell depletion studies, Abs to deplete CD4 (clone GK1.5; BioXcell) or CD8 (clone 2.43; BioXCell) were injected intraperitoneally at $100 \mu \mathrm{g}$ per mouse on days $-2,-1,+1,+4$ and every other 3 days afterwards until completion of the study as previously described. ${ }^{27}$ For survival studies, KPC-Brca2 mice were treated starting at 5 weeks of age with isotype control, IL-6 or PD-L1 Abs as single agents or in combination $(200 \mu \mathrm{g} /$ mouse each $\mathrm{Ab}$, BioXCell) until mice were moribund as determined by institutional animal care and use committee (IACUC) protocol.

\section{PSC isolation and Nanostring analysis}

Tissue from human patient pancreatic tumours undergoing surgical resection at the James Cancer Hospital and Solove Research Institute (Columbus, Ohio, USA) was obtained under an institutional review board-approved protocol following informed consent. Tissue was dissected with a scalpel into $0.5-1 \mathrm{~mm}^{3}$ pieces, and then plated in six-well $10-\mathrm{cm}^{2}$ uncoated culture wells in DMEM with $10 \%$ FBS and antibiotics and incubated at $37^{\circ} \mathrm{C}$. PSCs typically grew out of the tissue in 2-3 weeks and were characterised by morphology and histological analysis of $\alpha$-smooth muscle actin $\left(\alpha-\mathrm{SMA}^{+}\right)$staining. PSCs were maintained in culture with fresh media added twice weekly for three passages and then
RNA was collected by Trizol extraction. RNA was analysed using the nCounter PanCancer Immune Profiling Panel (Nanostring Technologies, Seattle, Washington, USA).

\section{Immunohistochemical analysis}

Tumour tissue was prospectively obtained at surgery under an institutional review board-approved protocol following informed consent and deidentified. Formalin-fixed pancreatic tissue from human tissue specimens and in vivo experiments in mice were subjected to immunohistochemical (IHC) analysis following staining with Ab against IL-6 (Abcam and eBioscience), pSTAT3 (Catalog 4904; Cell Signaling), $\alpha$-SMA (Catalog M0851; Dako), PD-L1 (Catalog NBP1-76769) and CD3 (Catalog A0452; Dako). For $\alpha$-SMA quantification, $20 \times$ magnification images of pancreata (8-10 images per mouse) were captured using PerkinElmer's Vectra multispectral slide analysis system. inForm software tools were used to quantify positive SMA cells (Fast Red chromogen) within each image (see online supplementary figure 1). For analysis of $\mathrm{CD}^{+}$cells, blinded histological analysis of staining in the pancreas was counted at $40 \times$ magnification, with at least 10 fields counted per mouse.

\section{Flow cytometry}

Immunophenotypic analyses of splenoctyes and single cell suspensions from tumours were assessed by flow cytometry. Abs to stain for MDSCs were CD11b-APC (clone M1/70; BD
Figure 1 Profile of mRNA transcripts from patient-derived pancreatic stellate cells (PSCs). RNA was isolated from 10 patient-derived pancreatic cancer stellate cells and analysed using the Nanostring nCounter PanCan Immune Profiling Panel. Data are expressed as the fold-change in expression as compared with a normal human pancreatic fibroblast cell line and relative to several housekeeping genes. Genes are ranked from highest fold-change to lowest. Heat map with hierarchical clustering for genes with at least twofold change up or down with a $p<0.01$ cut-off. Significantly higher expression is shown in red and lower expression in green.

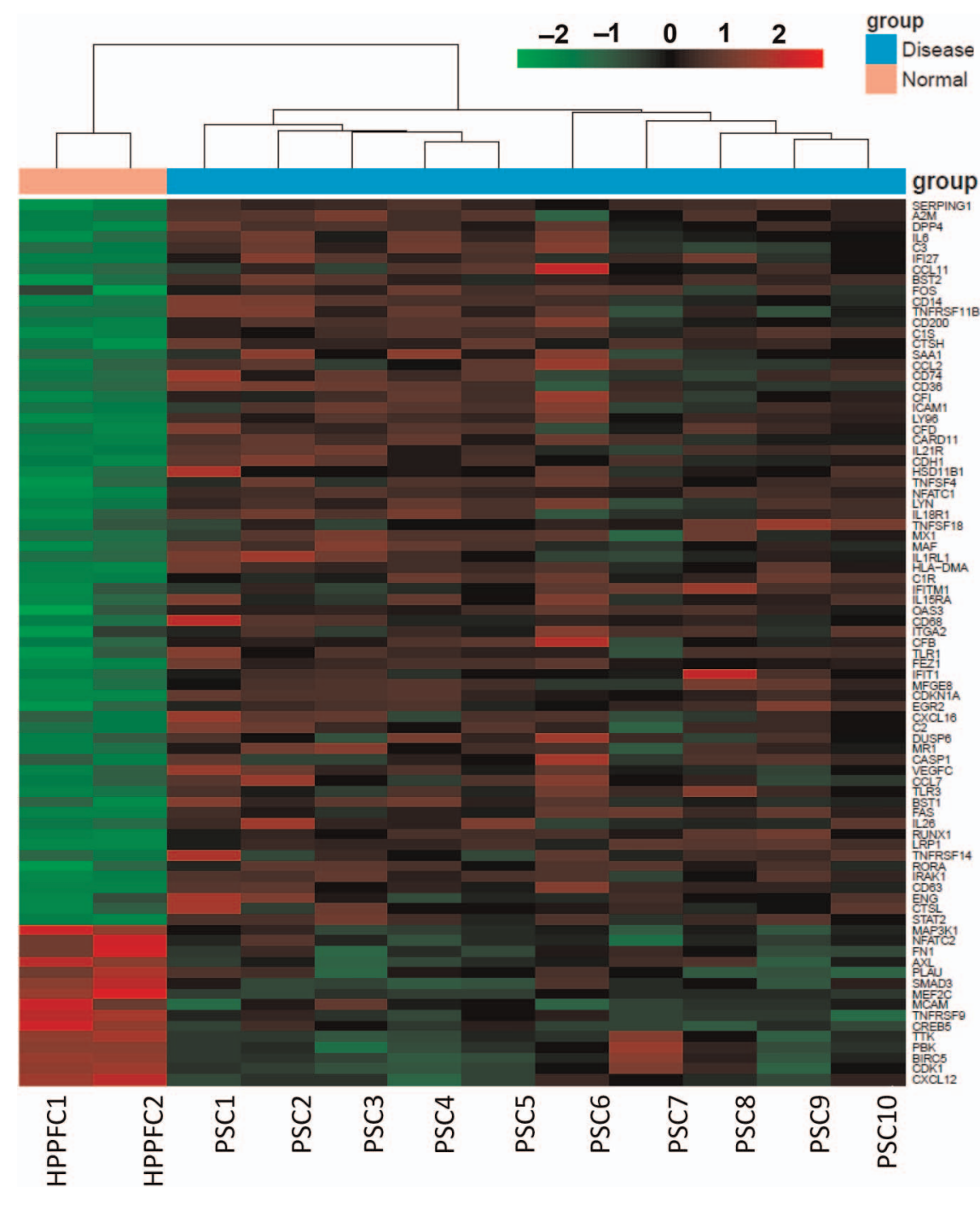

Mace TA, et al. Gut 2018;67:320-332. doi:10.1136/gutjnl-2016-311585 
Biosciences), Ly6G-FITC (clone 1A8; BD Biosciences) and Ly6C-PE (clone AL-21; BD Biosciences). For T-cell activation markers, cells were stained with $\mathrm{Ab}$ specific for CD4-PE-Cy7 (clone RM4-5; BD Biosciences), CD8-PE-Cy7 (clone 53-6.7; BD Biosciences), CD62L-PE (clone MEL-14; BD Biosciences) and CD44-FITC (clone IM7; Biolegend). To determine Th1 and Th2 phenotypes, cells were stained using fluorochrome-conjugated Ab-targeted CXCR3-PE-Cy7 (clone CXCR13-173; Biolegend), CCR4-PE (clone 2G12; Biolegend) and CCR6-APC (clone CK4-L3; BD Biosciences). Cells were incubated on ice for $30 \mathrm{~min}$, washed and fixed in phosphate buffered saline (PBS) containing 1\% formalin for flow cytometric analysis on a LSRII flow cytometer (BD Biosciences).

\section{Statistics}

For data obtained by Nanostring (Nanostring Technologies, Seattle, Washington, USA), positive spike-in controls were first used to normalise assay efficiency. Negative controls were used to access background hybridisation and for filtering out low expression probes. Reference genes were used to normalise across biological samples. Linear mixed models were then used to detect differentially expressed RNAs. A variance smoothing method was used for improving variance estimates in testing. ${ }^{28}$ Significance was adjusted by controlling the mean number of false positives. ${ }^{29}$ Data obtained by flow cytometry and IHC as well as tumour volumes were log-transformed prior to analysis to meet model assumptions of normality and homoscedasticity. Poisson regression was used to model the number of PanIN3/ tumour lesions in the KPC-Brca2 mice. Tumour volume was modelled over time using mixed-effects regression with fixed effects for group, time and the interaction of the two. Random intercepts and slopes by mouse were included with an unstructured covariance matrix for the random effects. For the T-cell depletion experiments examining tumour growth, interaction contrasts were constructed to enable the direct testing of the hypotheses under consideration. ${ }^{30}$ For Kaplan-Meier survival
A
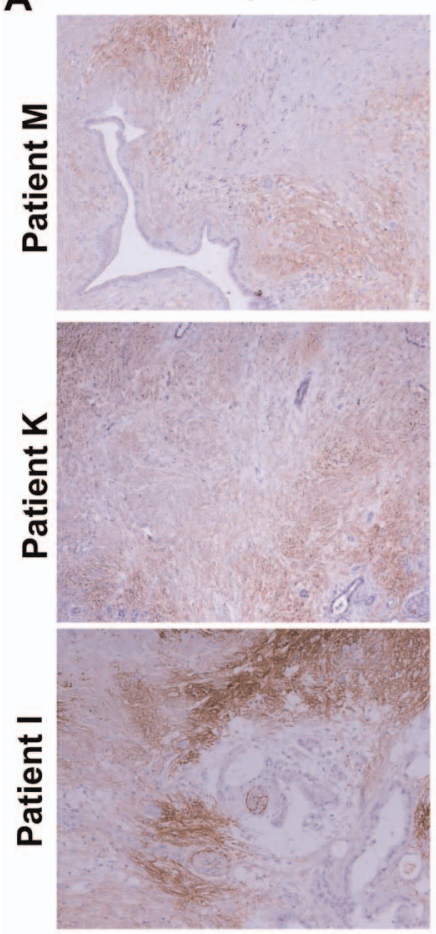

IL-6 (20x)
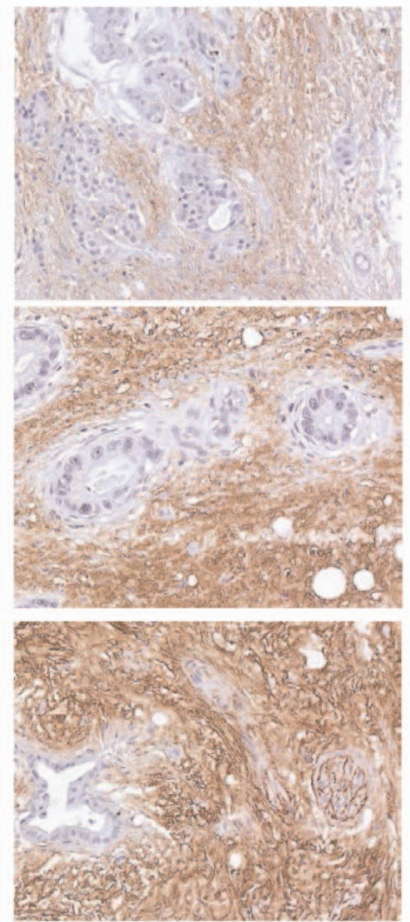

Isotype control (20x)
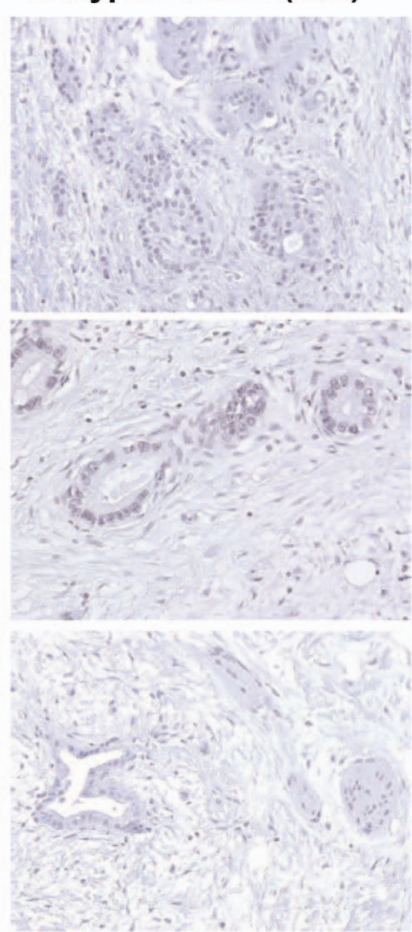

B

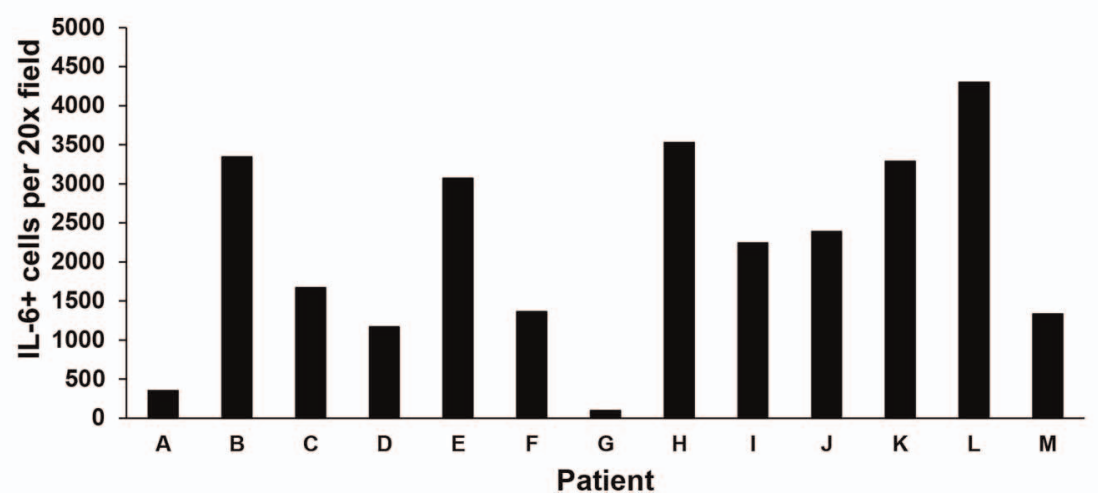

Figure 2 Interleukin 6 (IL-6) expression is enriched in the stromal compartments of human pancreatic ductal adenocarcinoma (PDAC) tissue. Human PDAC tumours (among $n=13$ patients) stained for IL-6 expression by immunohistochemistry. (A) IL-6 (Brown Chromogen) immunohistochemical (IHC) staining from three representative patient samples is displayed at $10 \times$ and $20 \times$ magnification. Tissue sections from each patient were stained with an isotype control Ab to account for background. (B) Number of IL-6-positive cells per field was quantified. 
curves, a log-rank test to determine difference used. The other outcomes were compared using ANOVA. p Values $<0.05$ were considered significant and all analyses were conducted in SAS V.9.4 (SAS Institute, Cary, North Carolina, USA) or R V.3.2.3 (R Foundation for Statistical Computing, Vienna, Austria).

\section{RESULTS}

The pancreatic tumour stroma is a major source of IL-6 in the tumour microenvironment

For these studies, an unsupervised assessment of gene expression was conducted to identify actionable immunological targets in PSCs, a prominent component of PDAC stroma. Stellate cells derived from surgically resected pancreatic tissue of 10 different patients were isolated and cultured in vitro as previously described. ${ }^{14}$ After three passages, RNA was extracted and subjected to profiling of transcripts relevant to immunological biomarkers using Nanostring technology (Nanostring PanCancer Immune Profiling Panel). RNA isolated from patient-derived PSCs exhibited significantly increased expression of cytokines and chemokines including IL-6 and CCL11 as compared with RNA isolated from normal human pancreatic fibroblasts (figure 1 and online supplementary figure 2). Further investigation via IHC analysis confirmed a pattern of staining for IL-6 that localised predominantly in the desmoplastic, stromal regions of pancreatic tumours, with occasional staining in regions containing malignant pancreatic epithelial cells (figure 2A). This trend was evident in tissue from several $(n=13)$ representative patients (figure $2 B)$. These data were consistent with our analysis of transcripts in PSCs and suggested that the stromal compartment, is a major source of IL-6 in the pancreatic tumour microenvironment.

\section{Prominent expression of IL-6/JAK/STAT pathway components and PD-L1 in the pancreatic tumour microenvironment of mouse models of pancreatic cancer}

Similar to human PDAC, histological characterisation of pancreata from PDAC orthotopic and GEMM revealed expression of IL-6 in both stroma, and more limited positive staining in foci of some tumours (figure 3). Consistent with prior published studies, pancreata from GEMM (KPC and KPC-Brca2) recapitulated the histological features of advanced PDAC observed in human disease. For example, KPC tumours displayed acinar to ductal metaplasia, while the KPC-Brca2 displayed desmoplastic stroma, PanIN and ductal adenocarcinoma expression similar to observations in human tissue. Pancreata from both the orthotopic and genetic tumour models displayed a fibrotic stroma that both surrounded and intercalated within tumour foci (figure 3; S, stroma; T, tumour tissue). Of note, robust phosphorylation of STAT3 was detectable in stroma and malignant epithelial cells in the pancreata from both KPC and KPC-Brca2 mice (figure 3). Consistent with this immunosuppressive microenvironment, tumours expressed high levels of PD-L1, regardless of the animal model.

\section{Combined blockade of IL-6 and PD-L1 inhibits murine pancreatic tumour growth and increases intratumoural effector T cells in vivo}

We postulated that concurrent blockade of IL- 6 and a potent immune-modulatory stimulus via PD-L1-targeting Ab would circumvent key mechanisms of resistance to T-cell-mediated immunity to pancreatic cancer. We used murine MT5 tumour cells subcutaneously injected into C57BL/6 mice. This cell line was derived from KPC tumours and has both G12D mutated Kras and R172H Trp53. ${ }^{31}$ Once tumours were palpable, mice were treated with anti-IL-6, anti-PD-L1 or isotype controls until study endpoint. A significant inhibition of tumour growth was observed in response to the combination therapy as compared with isotype, anti-IL-6 or PD-L1 Ab therapy alone (figure 4A; $\mathrm{p}<0.03)$. To carefully assess immune changes and confirm our data in an additional PDAC model, the well-characterised, murine Panc02 model was also used, given its frequent use in published preclinical immunotherapy studies in PDAC. Similar to the MT5 cell line, Panc02 cells are also syngeneic to immune competent C57BL/6 mice. ${ }^{32} 33$ Once tumours were palpable,

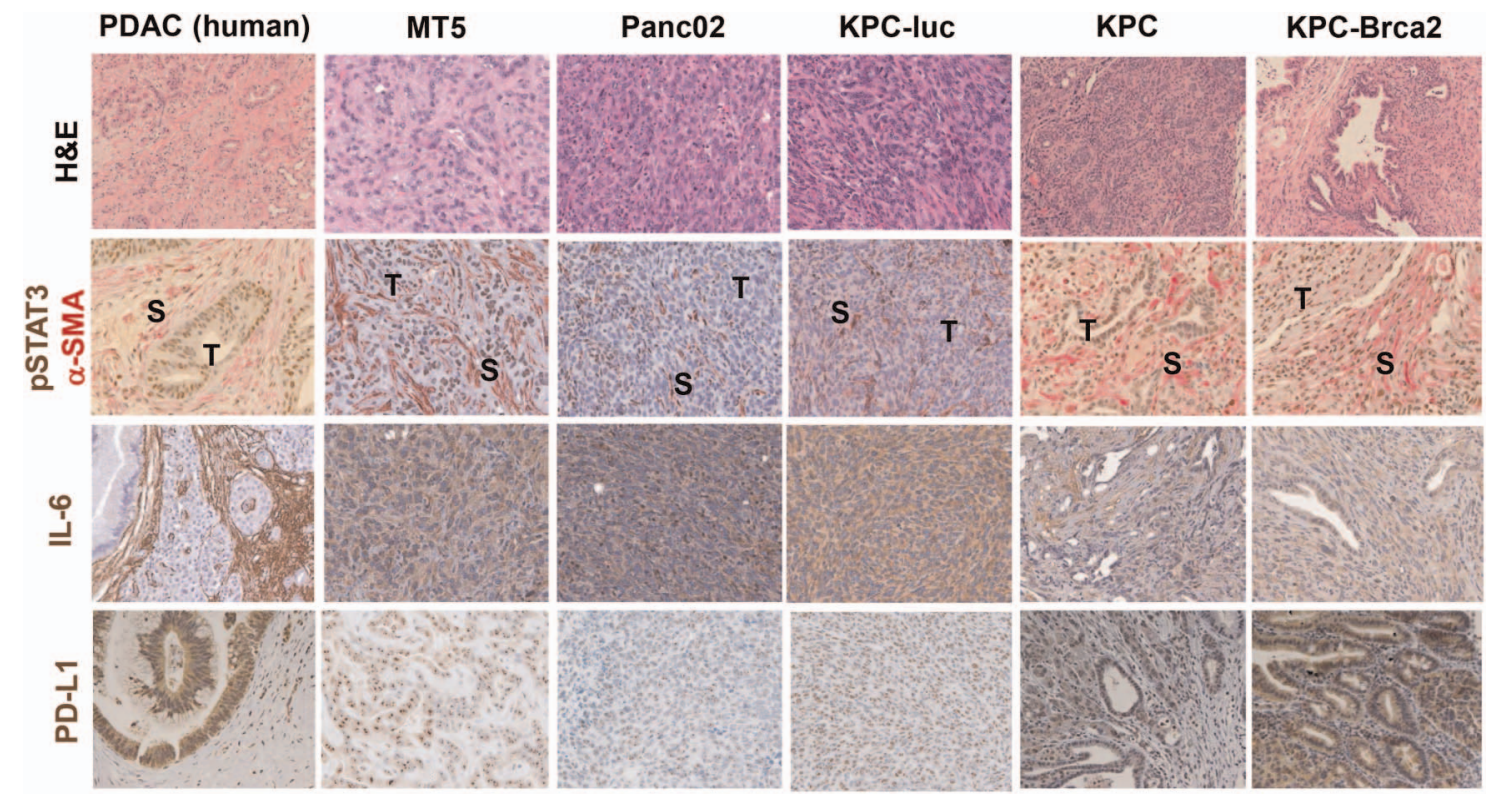

Figure 3 Pancreatic ductal adenocarcinoma (PDAC) genetically engineered mouse model (GEMM) recapitulate interleukin 6 (IL-6) pathway activation and PD-L1 expression observed in human disease. Immunohistochemical (IHC) analysis of representative H\&E, pSTAT3 (brown)/ $\alpha$-smooth muscle actin ( $\alpha-S M A)($ pink), IL-6 (brown) and PD-L1 (brown) staining on tissue sections from human pancreatic cancer, orthotopic (KPC-luc), subcutaneous (MT5, Panc02) or pancreata from the following GEMM: KPC (Kras ${ }^{\text {LSL-G12D }}$, Trp53 ${ }^{\text {LSL-R270H }}$, Pdx1-cre) and KPC-Brca2 (Kras ${ }^{\text {LSL-G12D }}$ $\left.\operatorname{Trp} 53^{\mathrm{LSL}-\mathrm{R} 270 \mathrm{H}}, \mathrm{Pdx1} 1-\mathrm{cre}, \mathrm{Brca} 2^{\mathrm{F} / \mathrm{F}}\right)$. All images are presented at $40 \times$ magnification. 
mice received treatment until the study endpoint. A significant inhibition of tumour growth was observed in response to combined therapy with anti-IL- 6 and anti-PD-L1 Abs as compared with isotype controls (figure 4B; $\mathrm{p}=0.0457$ ). Tumours were dissociated at the study endpoint and underwent phenotypic analysis by flow cytometry. These data confirmed a significantly greater percentage of infiltrating $\mathrm{CD} 8^{+} \mathrm{T}$ cells in the tumour tissue from mice receiving the combination (figure $4 \mathrm{C}$; $\mathrm{p}=0.0006$ ). Further analysis indicated an increased proportion of cells with an effector $\mathrm{CD} 62 \mathrm{~L}^{-} \mathrm{CD} 44^{+}$phenotype in mice receiving combined blockade of IL-6 and PD-L1 (figure 4D; $p=0.0086$ and figure $4 \mathrm{E} ; \mathrm{p}=0.0024)$. Further, we observed no difference in the level of other circulating or intratumoural immunosuppressive subsets (granulocytic and monocytic MDSCs) between any of the treatment groups as compared with isotype controls (see online supplementary figure $3 \mathrm{~A}-\mathrm{C}$ ). Importantly, treatment Ab blocking IL-6, PD-L1 or both Ab combined did not result in any overt signs of toxicity as evidenced by weight loss in all animal models tested (data not shown).

\section{Decreased tumour growth by combined blockade of IL-6 and PD-L1 is CD8 ${ }^{+}$T-cell dependent}

To determine whether the efficacy of combined IL-6 and PD-L1 blockade was T-cell dependent, Panc02-bearing mice were administered $\mathrm{CD}^{+}$or $\mathrm{CD}^{+}$depleting Abs during the course of treatment. T-cell depletion was confirmed by flow cytometry

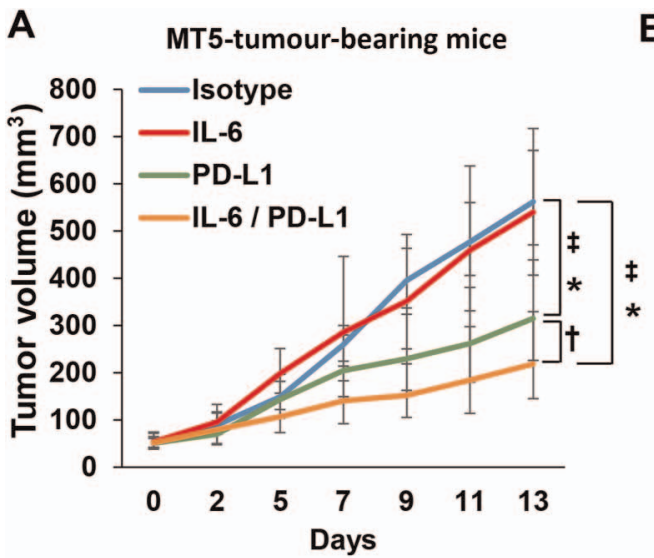

B
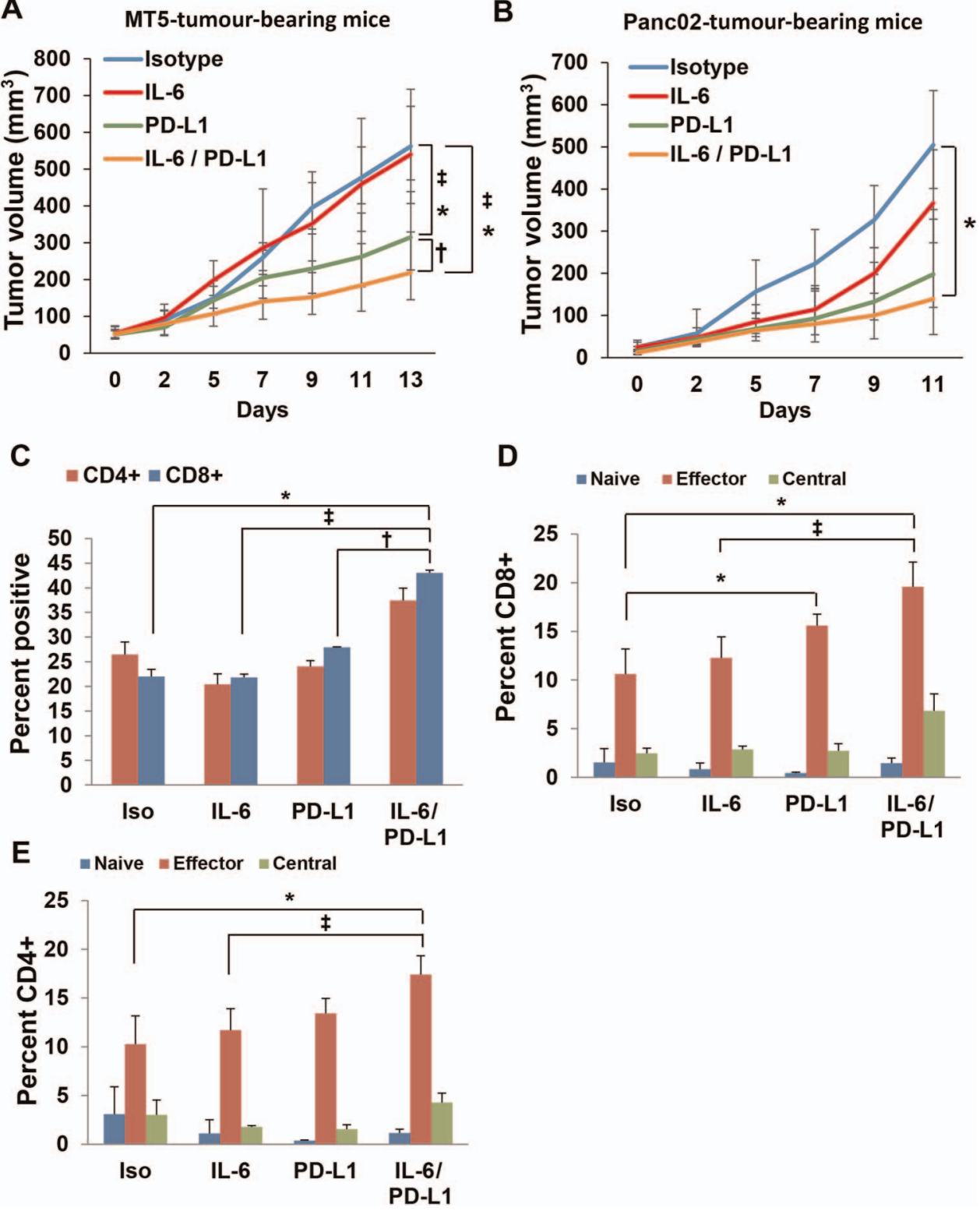

Figure 4 Interleukin 6 (IL-6) and programmed death-1-ligand 1 (PD-L1) antibody blockade combination therapy decreases pancreatic ductal adenocarcinoma (PDAC) tumour progression and increases the percentage of intratumoural effector T cells. (A) MT5 or (B) Panc02 murine pancreatic tumour cells were subcutaneously injected into C57BL/6 mice with treatment beginning when tumours reached $50-100 \mathrm{~mm}^{3}$. Mice were treated with $200 \mu \mathrm{g}$ (intraperitoneal injection three times/week) with isotype control, anti-IL-6 and/or anti-PD-L1 antibodies ( $\mathrm{n}=5-6 \mathrm{mice} / \mathrm{group}$ ) until mice met prespecified IACUC-approved early removal criteria. Geometric means $\pm S D$; * $p<0.01$ compared with isotype; $† p<0.03$ compared with PD-L1; $\neq p<0.05$ compared with IL-6. Panc02 tumours were dissociated using collagenase II and the Miltenyi Biotec gentleMACS dissociator to obtain a single-cell suspension. Cells were stained and analysed by flow cytometry for (C) CD4 ${ }^{+}, \mathrm{CD}^{+} \mathrm{T}$ cells and activation markers for different (D) CD4 ${ }^{+}$ and (E) CD8 ${ }^{+}$T-cell subsets ( $n=3 /$ group). Naïve $\left(C D 62 L^{+} C D 44^{-}\right)$, effector memory $\left(C D 62 L^{-} C D 44^{+}\right)$and central memory $\left(C D 62 L^{+} C D 44^{+}\right)$. Means $\pm S D$; ${ }^{*} \mathrm{p}<0.002$ compared with isotype; $\mathrm{tp}<0.01$ compared with PD-L1; $\neq \mathrm{p}<0.02$ compared with IL-6. 
using splenocytes from mice at the study endpoint (figure $5 \mathrm{~A}$, B). Administration of CD8 $(p=0.0016)$ but not CD4 depleting Abs abrogated the efficacy of combined IL-6 and PD-L1 blockade in mice bearing Panc02 tumours (figure 5C).

\section{IL-6 and PD-L1 Ab blockade combination therapy reduces tumour progression in an orthotopic model of PDAC}

To further investigate the efficacy of IL- 6 blockade combined with PD-L1 inhibition, we used KPC-luc cells orthotopically injected into the tail of the pancreas of C57BL/6 mice. This luciferase-expressing cell line was derived from KPC tumours with G12D-mutated Kras and R172H Trp53. ${ }^{25}$ Tumour growth was assessed by weekly bioluminescent imaging (figure 6A,B). Once tumours were detectable by imaging the pancreas, mice were randomised and treated with anti-IL-6, anti-PD-L1 or isotype control Abs until the study endpoint. We observed a decreased final tumour weight in mice receiving combination therapy as compared with isotype control, anti-IL-6 or PD-L1 $\mathrm{Ab}$ therapy alone (figure 6C; $\mathrm{p}=0.05530$ ). A decrease in $\alpha-\mathrm{SMA}^{+}$stromal cells was also observed in the pancreata of mice treated with combination therapy as compared with isotype control $\mathrm{Ab}$ alone $(\mathrm{p}=0.0563)$. Furthermore, the number of $\mathrm{CD}^{+}{ }^{+} \mathrm{T}$ cells was greater in the pancreata from mice treated with Abs targeting both IL-6 and PD-L1 as compared with those treated with isotype control Abs alone $(p=0.0553)$.

\section{Abs targeting IL-6R and PD-L1 limit tumour progression in the aggressive KPC-Brca2 GEMM}

To further confirm our data in a genetic model that better recapitulates many aspects of patient tumours, we used a highly aggressive, autochthonous model of spontaneously arising PDAC driven by mutants Kras, Trp53 and Brca2 (figure 7A). Any efficacy in this oncogene-driven tumour model would signify a promising approach to carry forward for further evaluation. At 5-6 weeks of age, KPC-Brca2 mice have PDAC with $100 \%$ penetrance (figure $7 \mathrm{~A}$ ). Following 2 weeks of treatment with anti-IL-6R and/or anti-PD-L1, animals were euthanised and pancreata was isolated for histological analysis. There was a statistically significant shift in the proportion of low-grade PanIN lesions, with fewer PanIN3 or foci of adenocarcinoma in mice treated with Abs targeting PD-L1 and IL-6R, as compared with animals treated with isotype control $\mathrm{Ab}$ (figure 7A, B; $\mathrm{p}<0.001)$. Similar to the subcutaneous Panc02 model, depletion of $\mathrm{CD} 8^{+} \mathrm{T}$ cells limited the effect of combination therapy in the
Figure 5 The antitumour response to combined interleukin 6 (IL-6) and programmed death-1-ligand 1 (PD-L1) blockade is $\mathrm{CD}^{+} \mathrm{T}$-cell-dependent. Panc02 murine pancreatic tumour cells were subcutaneously injected into C57BL/6 mice with treatment beginning when tumours reached 50 $100 \mathrm{~mm}^{3}$. Mice were also depleted of either $\mathrm{CD}^{+}$or $\mathrm{CD}^{+} \mathrm{T}$ cells by injecting depletion antibodies on days 5, 6, 8, 11 and 14. (A) Representative flow cytometry and (B) quantification of CD4 and CD8 staining of splenocytes to confirm depletion. Means $\pm S D ;{ }^{*} p<0.001$. (C) On day 7 , mice were treated with $200 \mu \mathrm{g}$ (intraperitoneal injection three times/ week) of isotype controls or anti-IL-6 and anti-PD-L1 antibodies combined ( $n=5$ mice/group) until mice met prespecified IACUC-approved early removal criteria. Means $\pm S D ;{ }^{*} p<0.001$ compared with isotype; $t p<0.002$ CD8 depletion versus CD4 depletion.
A Isotype

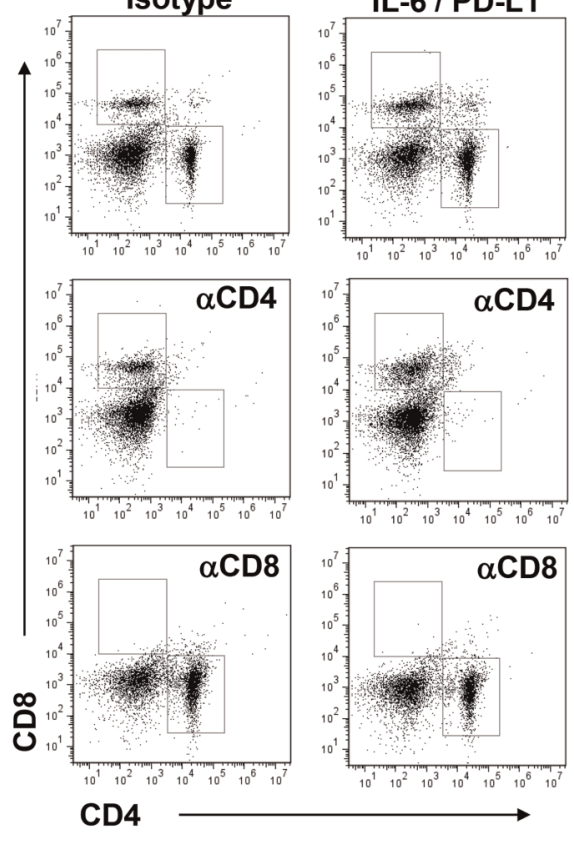

B

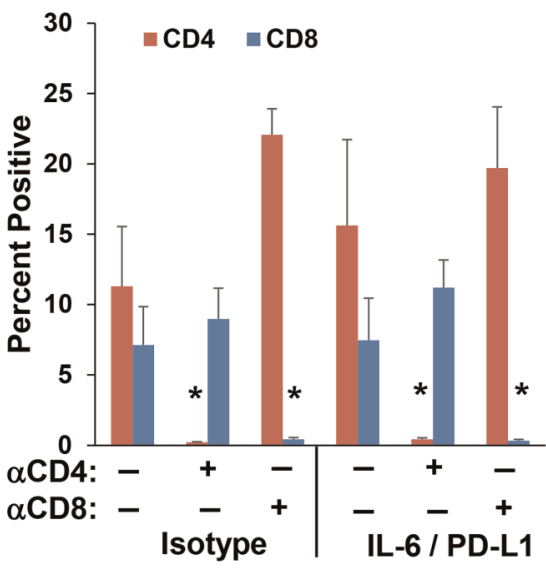

C

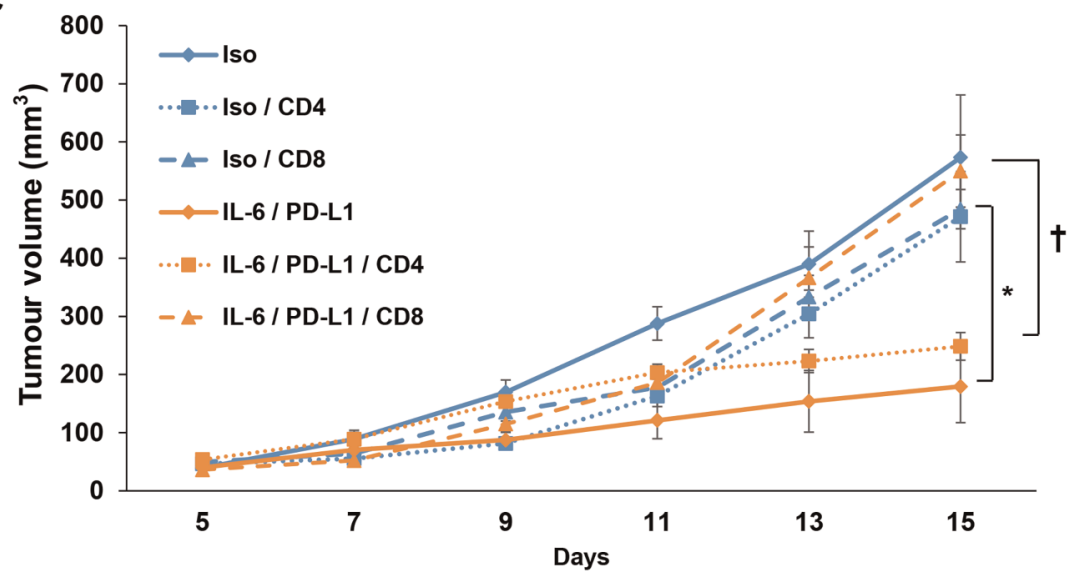


KPC-Brca2 GEMM. In fact, we observed a greater proportion of PanIN3/PDAC lesions in pancreata from $\mathrm{CD} 8^{+}$-depleted mice treated with the combination when compared with mice treated with the combination alone (figure 7B). Importantly, analysis revealed a significant decrease in the number of $\alpha-\mathrm{SMA}^{+}$stromal cells in pancreata of mice treated with $\mathrm{Ab}$ targeting both IL-6R and PD-L1 as compared with control mice (figure 7C,D; $\mathrm{p}<0.05$ ). Mice depleted of $\mathrm{CD} 8^{+} \mathrm{T}$ cells treated with combination therapy did not display the same decrease of $\alpha-\mathrm{SMA}^{+}$cells in the pancreas compared with mice treated with only the combination therapy (figure 7D; $p=0.0033$ ). Further, treatment with Ab targeting IL-6, PD-L1 or both agents combined did not alter expression of IL-6 (see online supplementary figure 5A) or PD-L1 (see online supplementary figure 5B) in the pancreatic tumour microenvironment.

Combined blockade of IL-6R and PD-L1 increases T cells with Th1 phenoytpic properties and $\mathrm{CD}^{+}$cell infiltration into PDAC tumours in KPC-Brca2 mice

To better understand the mechanism by which combined IL-6 and PD-L1 blockade limited tumour progression in the aggressive KPC-Brca2 model, a series of immunophenotypic analyses were conducted. These data revealed a higher percentage of circulating $\mathrm{CD}^{+} \mathrm{T}$ cells with $\mathrm{Th} 1$ phenotypic properties $\left(\mathrm{CXCR} 3^{+} \mathrm{CCR}^{-}{ }^{-} \mathrm{CCR} 6^{-}\right)$in the spleens of mice treated with $\mathrm{Ab}$ targeting IL-6R and PD-L1 combined as compared with

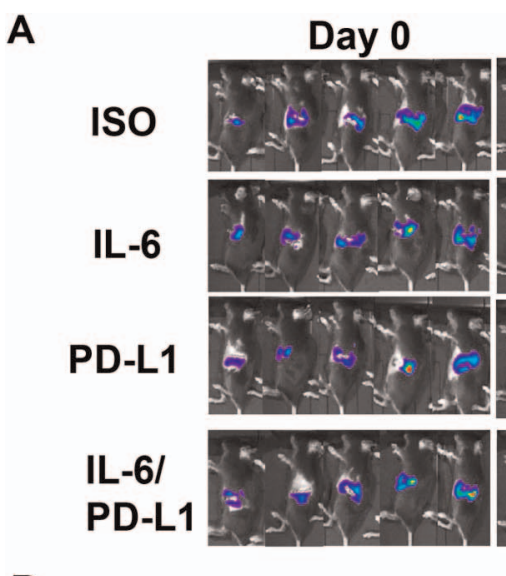

B
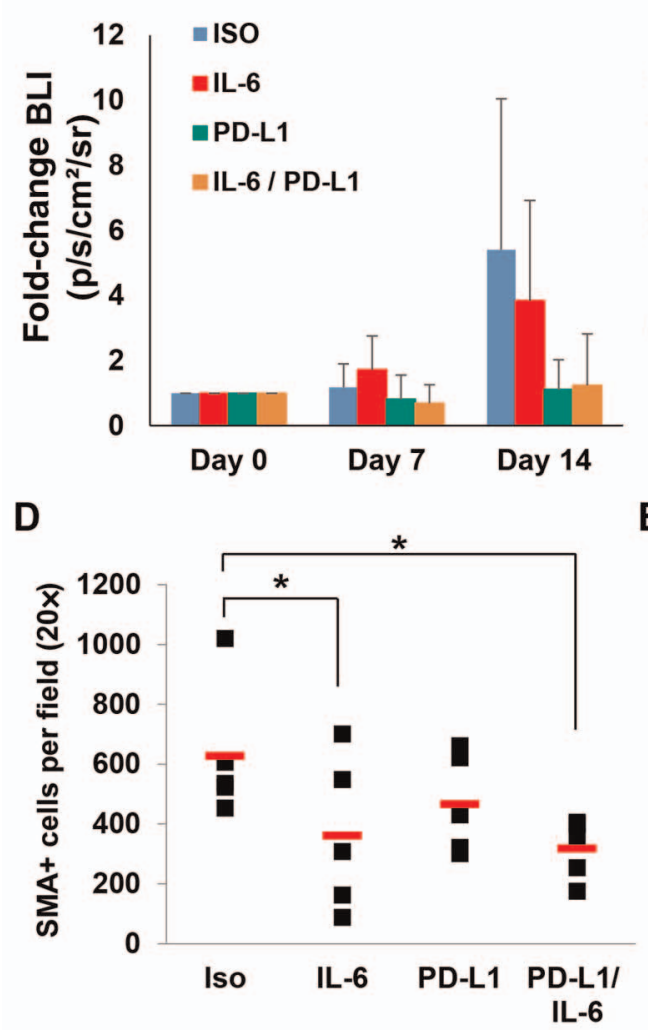

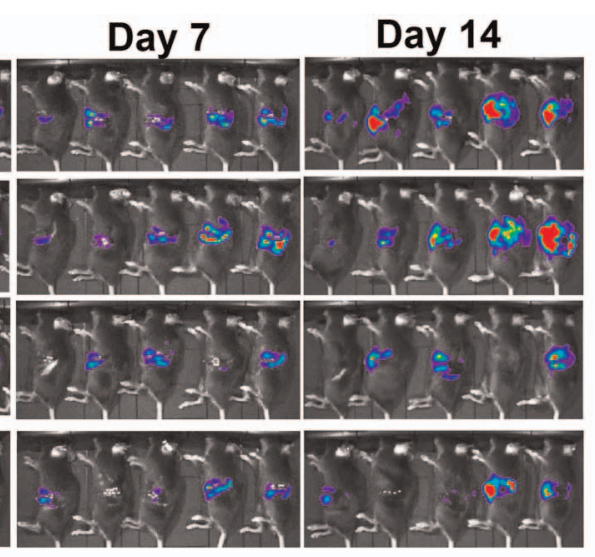

C
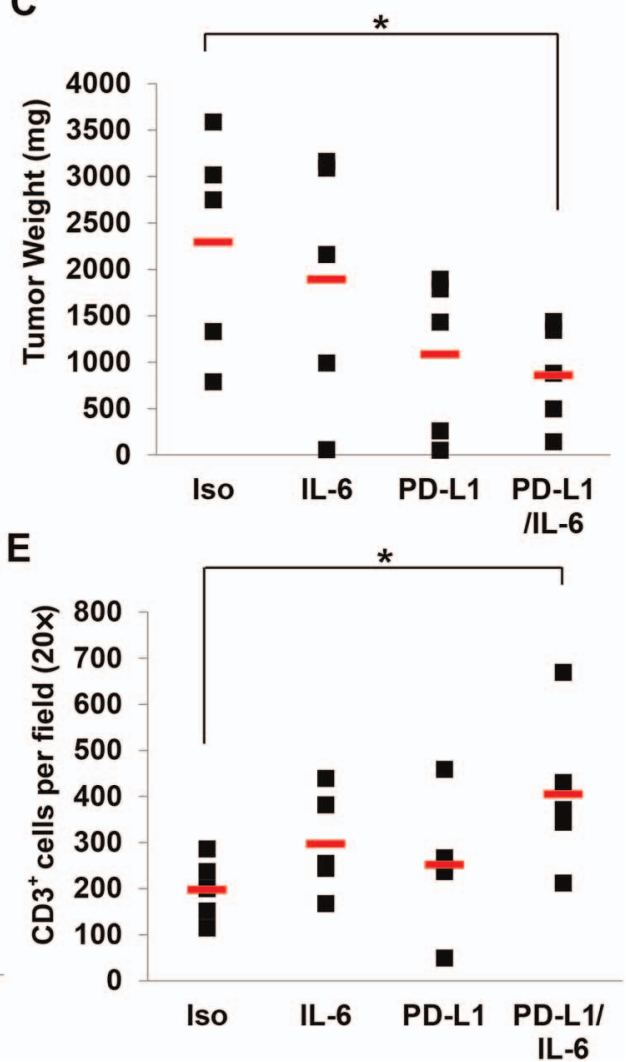

Figure 6 Interleukin 6 (IL-6) and programmed death-1-ligand 1 (PD-L1) antibody combination blockade reduces tumour growth in an orthotopic model of pancreatic cancer. C57BL/6 mice were orthotopically injected with luciferase-expressing KPC cells (KPC-luc) in matrigel and (A) tumour growth assessed and (B) quantified by bioluminescent imaging weekly. (C) Tumour weights at completion of study. Means $\pm S D$; ${ }^{*} \mathrm{p}=0.05530$ compared with isotype. Pancreatic tissue was stained for (D) $\alpha$-smooth muscle actin ( $\alpha$-SMA+) stromal cells (means $\pm S D ;{ }^{*} p=0.0563$ compared with isotype) and (E) $\mathrm{CD}^{+} \mathrm{T}$ cells (means $\pm \mathrm{SD} ;{ }^{*} \mathrm{p}=0.0553$ compared with isotype) by immunohistochemical (IHC) analysis quantified at $20 \times$ magnification. 
controls (figure $8 \mathrm{~A} ; \mathrm{p}<0.0001$ ). No changes in $\mathrm{CD}^{+} \mathrm{T}$ cells with Th2 phenotypic properties were evident in any treatment group as compared with controls (figure 8B). Importantly, assessment of the tumour microenvironment revealed significant increases in the infiltrating $\mathrm{CD}^{+} \mathrm{T}$ cells in the pancreata of combination treated mice (figure $8 \mathrm{C}, \mathrm{D} ; \mathrm{p}=0.0025$ ). Further analysis revealed no difference in the level of other immunosuppressive subsets (MDSCs and Tregs) between any of the treatment groups as compared with isotype controls (see online supplementary figure $4 \mathrm{~A}-\mathrm{D})$. These data suggest that this treatment regimen may modulate phenotypic properties of $\mathrm{T}$ cells and their access to the tumour microenvironment.

\section{Combined blockade of IL-6 and PD-L1 extend overall survival in the KPC-Brca2 GEMM}

The impact of this treatment combination on overall survival was evaluated in KPC-Brca2 mice. In this study, mice began
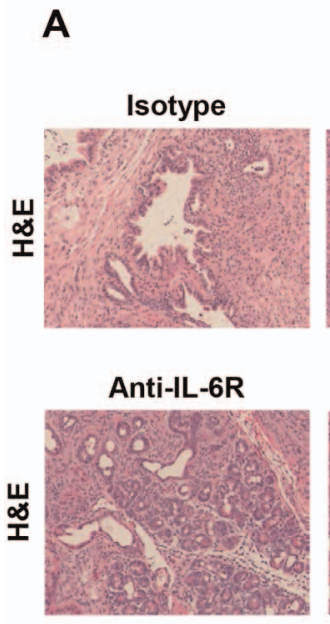

C
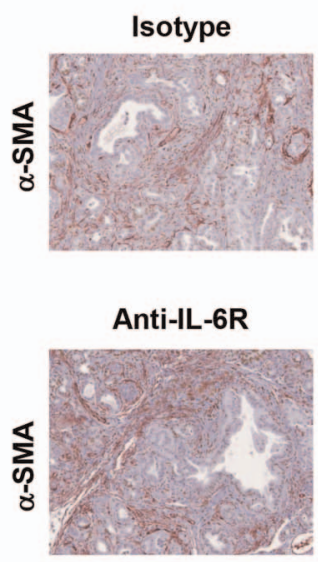

Anti-PD-L1

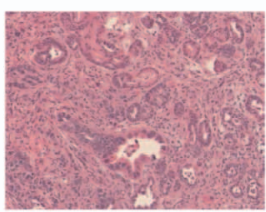

Anti-PD-L1 + Anti-IL-6R

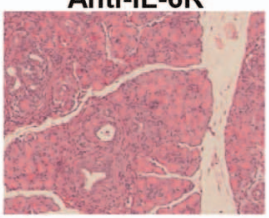

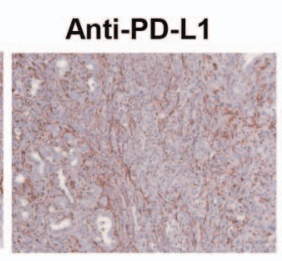

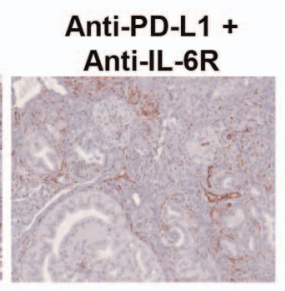

B

In the present report, we demonstrate that combined blockade of IL-6 and PD-L1 elicits efficacy and extends survival therapy in highly aggressive PDAC models. These data extend prior observations regarding IL- 6 as a factor limiting the efficacy of immunotherapy in PDAC. Combination therapy skewed T cells

to a Th1 phenotype and increased the number of infiltrating

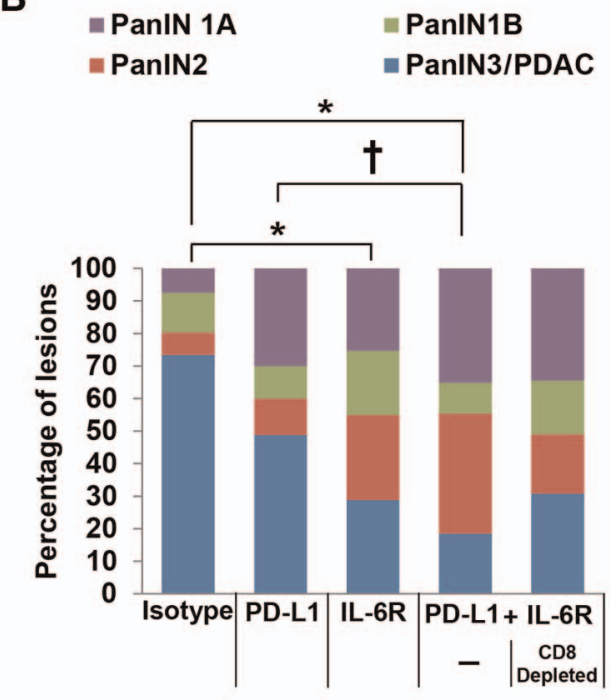

D

long-term treatment at 5 weeks of age with Abs targeting IL-6 and PD-L1 or isotype controls three times weekly until the animals were moribund. A significant prolongation in overall survival $(35 \% ; \mathrm{p}=0.0012)$ was observed in mice treated with combined IL-6 and PD-L1 Abs, as compared with isotype control treated animals (figure 9). Long-term exposure to this treatment regimen did not lead to alterations in body weight of these animals, indicating its tolerability during a sustained period of administration (see online supplementary figure 6).

\section{DISCUSSION}

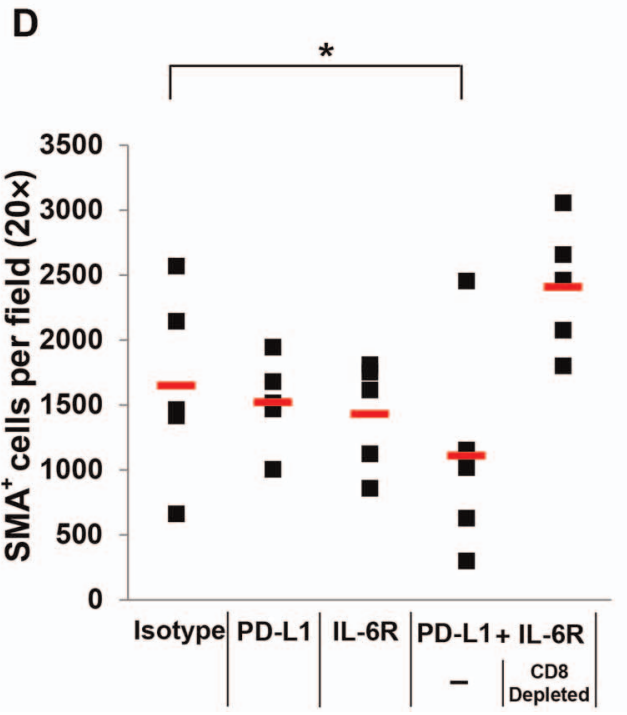

Figure 7 Interleukin 6 (IL-6) and programmed death-1-ligand 1 (PD-L1) antibody blockade combination therapy decreases pancreatic ductal adenocarcinoma (PDAC) tumour progression and $\alpha$-smooth muscle actin $\left(\alpha-S M A^{+}\right)$cells in the pancreata from KPC-Brca2 mice. KPC-Brca2 mice were treated at 5-6 weeks of age with $200 \mu \mathrm{g}$ (intraperitoneal injection three times/week) of isotype control, anti-IL-6R and/or anti-PD-L1 antibodies for 2 weeks ( $n=5$ mice/group). A cohort of mice treated with anti-IL-6R and PD-L1 antibodies were also depleted for CD8 ${ }^{+} T$ cells. (A) Representative $\mathrm{H} \& \mathrm{E}$ staining of pancreata at $20 \times$ magnification and (B) quantification of the pathology. Means $\pm S D ;{ }^{*} p<0.005$ compared with isotype; $\uparrow p=0.01$ compared with PD-L1; (C) Pancreatic tissue was stained for $\alpha$-smooth muscle actin ( $\alpha$-SMA+) stromal cells (red) by immunohistochemical analysis and (D) quantified at $20 \times$ magnification. Means $\pm S D ;{ }^{*} p=0.0545$ compared with isotype. 

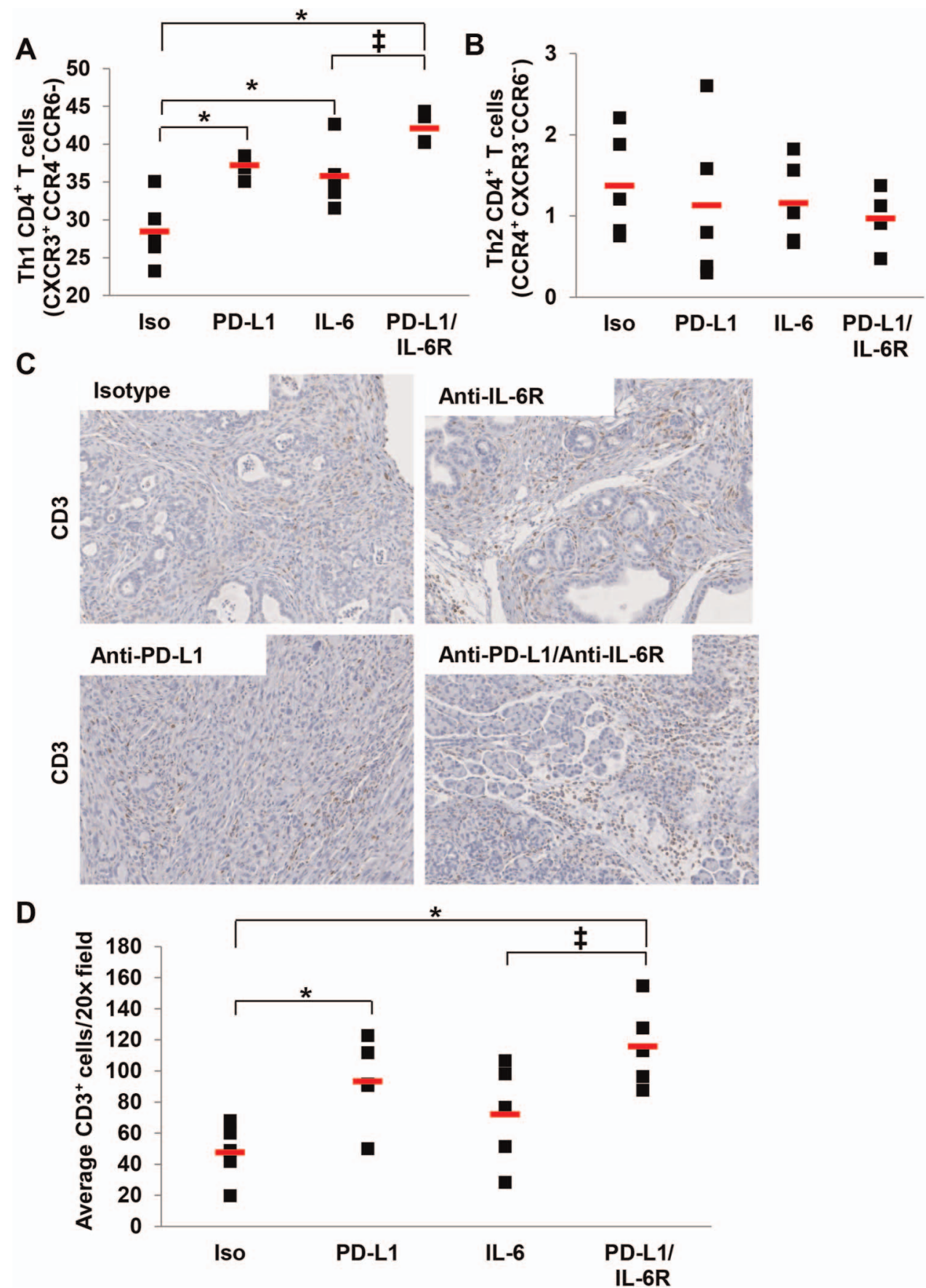

Figure 8 Higher percentage of circulating T cells with Th1 phenotypic properties and intratumoural CD3 $3^{+}$cells in mice treated with interleukin 6 (IL-6) and programmed death-1-ligand 1 (PD-L1) antibody blockade. KPC-Brca2 mice were treated at 5-6 weeks of age with $200 \mu \mathrm{g}$ (intraperitoneal injection three times/week) with isotype control, anti-IL-6R and/or anti-PD-L1 antibodies for 2 weeks ( $\mathrm{n}=5$ mice/group). Splenocytes were stained and analysed by flow cytometry for (A) T cells with Th1 phenotypic properties (CD4 ${ }^{+} \mathrm{CXCR} 3^{+} \mathrm{CCR} 4^{-} \mathrm{CCR} 6^{-}$) or (B) T cells with Th2 phenotypic

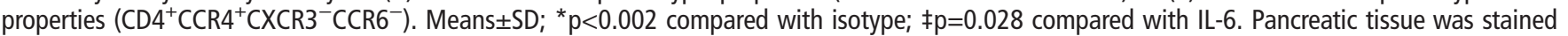
for (C) $\mathrm{CD}^{+} \mathrm{T}$ cells (brown) by immunohistochemical (IHC) analysis and (D) quantified at $20 \times$ magnification. Means $\pm S D ;{ }^{*} \mathrm{p}<0.05$ compared with isotype; $\neq p=0.052$ compared with IL-6.

effector cells into the tumour. This is significant, as limited T-cell infiltration is considered a barrier for efficacy of immunotherapy in PDAC. Further, we showed that the efficacy of combination therapy on tumour progression was dependent on $\mathrm{CD}^{+}$T cells in multiple models. Overall, these preclinical data support this combination as a rational, therapeutic approach to elicit improved T-cell-mediated immune responses to pancreatic cancer.
The next frontier of immunotherapy is discovering ways to enhance the efficacy of checkpoint inhibitors using combination approaches, especially in cancers where little effect is observed in the clinic. Before blindly moving forward with combination approaches, it will be important to prioritise therapies based on empirical data, and the relative importance of targeting each factor in the tumour-bearing host. IL-6 as a target is quite attractive for a number of reasons. For example, as recently reported by 


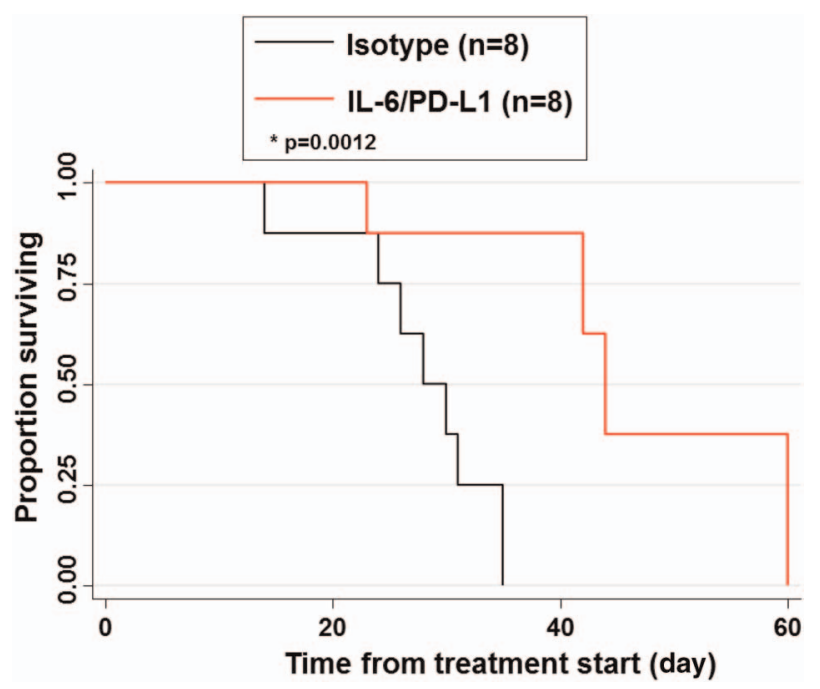

Figure 9 Interleukin 6 (IL-6) and programmed death-1-ligand 1 (PD-L1) blockade increases overall survival of KPC-Brca2 mice. KPC-Brca2 beginning at 5 weeks of age mice were treated with isotype control antibodies or antibodies targeting IL-6 and PD-L1 (200 $\mu \mathrm{g} / \mathrm{each})$ until mice were moribund and met prespecified IACUC-approved early removal criteria. Kaplan-Meier survival curves with log-rank test for significance between isotype control and IL-6/PD-L1 antibodies $(p=0.0012)$.

our group, higher baseline plasma IL-6 in treatment-naïve patients with PDAC correlated with worse overall survival. ${ }^{16}$ IL-6 can also promote the differentiation of dendritic cells (DCs), macrophages and MDSC contributing to immunosuppression. ${ }^{19} 22$ Further, IL-6 can lead to greater proliferation, invasiveness and tumour progression in the pancreatic tumour microenvironment. ${ }^{16}$ This report represents an important series of preclinical studies to provide the first evidence of this treatment combination working in relevant animal models. The next logistical step for effective translation will be to understand how this drug combination works in the context of prior or concurrent chemotherapy, which will be most relevant in clinical trial scenarios.

IL-6-targeting agents have been used in pancreatic cancer but with limited success. This is potentially because IL- 6 blockade has been attempted as a single agent, or in combination with chemotherapy in heavily pretreated patients. ${ }^{34} 35$ In all of these studies, there was no potent immunotherapeutic treatment that was given concurrently with blockade of IL-6. Others have shown that neutralisation of IL-6 also suppresses signalling through the PI3K/AKT pathway. ${ }^{36}$ A recent phase I study combining tocilizumab (anti-IL-6) and interferon (IFN)-a2b in ovarian cancer showed feasibility/safety and a greater percentage of activated T cells. ${ }^{37}$ Thus, blockade of IL- 6 could lead to potentially more efficacious results than targeting a single pathway (JAK/STAT, MEK and AKT) as it could simultaneously limit other pathways driven by aberrant IL-6 expression.

Our group and others have reported that tumours, myeloid cells and stromal cells can secrete IL-6, fuelling the tumour microenvironment and immunosuppression in PDAC. It is known that PDAC is profoundly immune suppressive, and clearly more data on clinical relevance of the stroma and its soluble factors, including IL-6, are needed. In a model of MCA205 cells transfected with OVA, Gr- $1^{+}$MDSCs were the source of IL-6 in tumour-bearing mice. Blockade of IL-6 led to increased differentiation of tumour-Ag-specific effector T helper cells (Th1). ${ }^{24}$ In this report, IL-6 in stellate cells derived from PDAC stroma was a key factor that emerged in an unsupervised analysis of patient PSCs at the RNA level and was confirmed via IHC. This was consistent with our prior in vitro data showing that patient-derived PSCs secrete IL- 6 that promotes the differentiation of MDSC. ${ }^{20}$ Other studies have clearly shown that IL-6 can arise from tumour-derived macrophages and may play a role via STAT3 in mediating immune suppression in the tumour microenvironment. ${ }^{5}$ These factors complement other cytokines such as GM-CSF, which is produced by tumour cells (and perhaps others) to fuel an immunosuppressive microenvironment. ${ }^{38}$ These data indicate that IL- 6 represents an actionable therapeutic target that may modulate the immune architecture in PDAC tumours and can be manipulated to limit immune suppression.

It is likely that the PDAC stroma amplifies immunosuppression via IL-6-induced signalling and limits the efficacy of immunotherapy. For instance, numerous reports suggest that a reciprocal relationship exists between tumour and stromal cells, leading to enhanced survival and growth of PDAC. ${ }^{39}$ Among these are studies demonstrating that fibroblast activating protein $\left(\mathrm{FAP}^{+}\right)$stromal cells maintain the stroma and suppress antitumour immunity. ${ }^{40-42}$ Other studies using targeted disruption of sonic hedgehog signalling in the stroma led to accelerated PDAC progression. ${ }^{43}$ These data provided insight into prior clinical studies using the hedgehog pathway inhibitor Saridegib, which were halted early due to patients progressing faster than those on the control arm. Similarly, genetic ablation of $\mathrm{SMA}^{+}$stromal cells led to accelerated metastasis and disease progression in a mutant KRas-driven model of PDAC. ${ }^{45}$ Despite these data, it was quite interesting to note that the response to immunotherapy with anti-CTLA4 $\mathrm{Ab}$ was enhanced in animals lacking $\mathrm{SMA}^{+}$stroma cells. In contrast, these animals did not derive benefit from chemotherapy with gemcitabine. ${ }^{45}$

Subsequent studies should investigate further how IL-6 blockade enhances checkpoint immunotherapy. IL-6 could act to promote expansion of MDSC, although no alterations were observed in MDSC levels in tumour and/or spleen in either the genetic or subcutaneous murine models (see online supplementary figures 3 and 4). MDSC and macrophages are other potential sources of IL-6, and blockade could also render these cells less immunosuppressive, leading to enhanced efficacy of checkpoint inhibition. IL- 6 could act at the level of antigenpresenting cells, by downregulating IL-12 production and subsequently the Th1 immune response. ${ }^{24} 46$ Another possibility is that IL-6 blockade could downregulate SOCS expression in T cells and restore the ability of IFN- $\gamma$ or other cytokines to act and promote Th1 differentiation. ${ }^{47}$ Although this is less likely given the redundancy in cytokines that might compensate and upregulate SOCS expression, it remains a possibility.

One final interesting and important observation was that anti-IL-6 and anti-PD-L1 combination increased T-cell infiltration into tumours. T-cell infiltration into tumours is a major issue that impacts the ability of a patient to respond to checkpoint inhibitors. ${ }^{48} 49$ This regimen clearly adjusts the phenotypic properties of $\mathrm{T}$ cells that infiltrate tumours and may make a subject more likely to respond to immunotherapy. Blockade of IL-6 in combination with checkpoint therapy could improve T-cell trafficking and change the profile of T cells in the tumour. We believe this combination may be a broadly applicable therapeutic strategy for multiple types of tumours that are driven by IL-6. Given the multiple facets of IL-6 in regulating pancreatic carcinogenesis and immune suppression, this represents a reasonable target with a rapid path to clinical translation since multiple IL- 6 blocking Abs are already FDA approved for other indications. 


\section{Author affiliations}

1 Divisions of Medical Oncology and Gastroenterology, Department of Internal Medicine, The Ohio State University, Columbus, Ohio, USA

${ }^{2}$ Comprehensive Cancer Center, The Ohio State University, Columbus, Ohio, USA

${ }^{3}$ Department of Molecular Virology, Immunology, and Medical Genetics, The Ohio State University, Columbus, Ohio, USA

${ }^{4}$ Department of Pathology, The Ohio State University, Columbus, Ohio, USA

${ }^{5}$ Department of Biomedical Informatics, The Ohio State University, Columbus, Ohio, USA

${ }^{6}$ Center for Biostatistics, The Ohio State University, Columbus, Ohio, USA

${ }^{7}$ Department of Surgery (Indiana University) and IU Simon Cancer Center, The Ohio State University, Columbus, Ohio, USA

${ }^{8}$ Division of Surgical Oncology, Department of Surgery, The Arthur G. James Cancer Hospital and Richard J. Solove Research Institute, The Ohio State University, Columbus, Ohio, USA

${ }^{9}$ Mayo Clinic, 5777 E. Mayo Blvd, Phoenix, Arizona, USA

${ }^{10}$ Department of Hematology and Medical Oncology, Winship Cancer Institute of Emory University, Atlanta, Georgia, USA

Acknowledgements We thank the OSU Comprehensive Cancer Center Biostatistics, Genomics, Target Validation, Analytical Cytometry and Comparative Pathology \& Mouse Phenotyping Shared Resources supported in part by National Cancer Institute grant P30 CA016058.

Contributors TAM, ZC-M, MCO, MB, TB-S and GBL contributed to conception and design of the study. TAM, RS, CWM, SL, EN and XZ performed experiments and acquired data. RS, ZC-M and TL provided expertise in genetic murine models and in vivo experiments. BS provided expertise in pathological analysis. MB provided patient surgical specimens and regulatory approval. GY provided expertise in statistical analysis. GY and LY provided expertise in bioinformatics. TAM, MCO, TB-S and GBL drafted the manuscript. TAM, JRP, GY, TAZ and GBL revised the manuscript critically for important intellectual content. All authors approved the final version of the manuscript.

Funding NIH Grants 5T32CA009338-34 (Mace), 1R21Al124687-01 (Lesinski), 1R01CA208253-01 (Lesinski), P30CA016058-36 (Caligiuri), American-Italian Cancer Foundation Pancreatic Cancer Initiative grant (Bloomston) and Lustgarten Foundation grant (Zimmers). The project described was supported by Award Number UL1RR025755 from the National Center for Research Resources, funded by the Office of the Director, National Institutes of Health (OD) and supported by the NIH Roadmap for Medical Research. Supported by the William Hall Fund for pancreatic and liver research. The project was described by Award Number Grant KL2TR001068 from the National Center for Advancing Translational Sciences. This work was also supported by the Pelotonia Fellowship Program. Any opinions, findings and conclusions expressed are those of the authors and do not necessarily reflect those of the Pelotonia Fellowship Program. The content is solely the responsibility of the authors and does not necessarily represent the official views of the National Center for Advancing Translational Sciences, the National Center for Research Resources or the National Institutes of Health.

Competing interests None declared.

Ethics approval The Ohio State University and Indiana University IRBs.

Provenance and peer review Not commissioned; externally peer reviewed.

\section{REFERENCES}

1 Rahib L, Smith BD, Aizenberg R, et al. Projecting cancer incidence and deaths to 2030: the unexpected burden of thyroid, liver, and pancreas cancers in the United States. Cancer Res 2014;74:2913-21.

2 Vincent A, Herman J, Schulick R, et al. Pancreatic cancer. Lancet 2011;378:607-20.

3 Al-Hajeili M, Azmi AS, Choi M. Nab-paclitaxel: potential for the treatment of advanced pancreatic cancer. Onco Targets Ther 2014;7:187-92.

4 Zhang Y, Yan W, Collins MA, et al. Interleukin- 6 is required for pancreatic cancer progression by promoting MAPK signaling activation and oxidative stress resistance. Cancer Res 2013;73:6359-74.

5 Lesina M, Kurkowski MU, Ludes K, et al. Stat3/Socs3 activation by IL-6 transsignaling promotes progression of pancreatic intraepithelial neoplasia and development of pancreatic cancer. Cancer Cell 2011;19:456-69.

6 Rose-John S, Scheller J, Elson G, et al. Interleukin- 6 biology is coordinated by membrane-bound and soluble receptors: role in inflammation and cancer. J Leukoc Biol 2006:80:227-36.

7 Fisher DT, Appenheimer MM, Evans SS. The two faces of IL- 6 in the tumor microenvironment. Semin Immunol 2014:26:38-47.

8 Scheller J, Chalaris A, Schmidt-Arras D, et al. The pro- and anti-inflammatory properties of the cytokine interleukin-6. Biochim Biophys Acta 2011;1813:878-88.

9 Corcoran RB, Contino G, Deshpande V, et al. STAT3 plays a critical role in KRAS-induced pancreatic tumorigenesis. Cancer Res 2011;71:5020-9.
10 Scholz A, Heinze S, Detjen KM, et al. Activated signal transducer and activator of transcription 3 (STAT3) supports the malignant phenotype of human pancreatic cancer. Gastroenterology 2003;125:891-905.

11 Gabitass RF, Annels NE, Stocken DD, et al. Elevated myeloid-derived suppressor cells in pancreatic, esophageal and gastric cancer are an independent prognostic factor and are associated with significant elevation of the Th2 cytokine interleukin-13. Cancer Immunol Immunother 2011;60:1419-30.

12 Markowitz J, Brooks TR, Duggan MC, et al. Patients with pancreatic adenocarcinoma exhibit elevated levels of myeloid-derived suppressor cells upon progression of disease. Cancer Immunol Immunother 2015;64:149-59.

13 Mundy-Bosse BL, Young GS, Bauer T, et al. Distinct myeloid suppressor cell subsets correlate with plasma IL- 6 and IL-10 and reduced interferon-alpha signaling in $\mathrm{CD}^{+} \mathrm{T}$ cells from patients with $\mathrm{GI}$ malignancy. Cancer Immunol Immunother 2011;60:1269-79.

14 Mace TA, Bloomston M, Lesinski GB. Pancreatic cancer-associated stellate cells: a viable target for reducing immunosuppression in the tumor microenvironment. Oncoimmunology 2013;2:e24891.

15 Goumas FA, Holmer R, Egberts JH, et al. Inhibition of IL-6 signaling significantly reduces primary tumor growth and recurrencies in orthotopic xenograft models of pancreatic cancer. Int J Cancer 2015;137:1035-46.

16 Farren MR, Mace TA, Geyer $\mathrm{S}$, et al. Systemic immune activity predicts overall survival in treatment naive patients with metastatic pancreatic cancer. Clin Cancer Res 2016;22:2565-74

17 Jiang X, Zhou J, Giobbie-Hurder A, et al. The activation of MAPK in melanoma cells resistant to BRAF inhibition promotes PD-L1 expression that is reversible by MEK and PI3K inhibition. Clin Cancer Res 2013;19:598-609.

18 Chen L, Han X. Anti-PD-1/PD-L1 therapy of human cancer: past, present, and future. J Clin Invest 2015;125:3384-91.

19 Freeman GJ, Long AJ, Iwai Y, et al. Engagement of the PD-1 immunoinhibitory receptor by a novel $B 7$ family member leads to negative regulation of lymphocyte activation. J Exp Med 2000;192:1027-34.

20 Goldberg MV, Maris CH, Hipkiss EL, et al. Role of PD-1 and its ligand, B7-H1, in early fate decisions of CD8 T cells. Blood 2007;110:186-92.

21 Topalian SL, Drake CG, Pardoll DM. Targeting the PD-1/B7-H1(PD-L1) pathway to activate anti-tumor immunity. Curr Opin Immunol 2012:24:207-12.

22 Duluc $D$, Delneste $Y$, Tan F, et al. Tumor-associated leukemia inhibitory factor and IL-6 skew monocyte differentiation into tumor-associated macrophage-like cells. Blood 2007;110:4319-30.

23 Marigo I, Bosio E, Solito $\mathrm{S}$, et al. Tumor-induced tolerance and immune suppression depend on the C/EBPbeta transcription factor. Immunity 2010;32:790-802.

24 Tsukamoto $\mathrm{H}$, Nishikata R, Senju S, et al. Myeloid-derived suppressor cells attenuate TH1 development through IL-6 production to promote tumor progression. Cancer Immunol Res 2013;1:64-76.

25 Ma Y, Hwang RF, Logsdon CD, et al. Dynamic mast cell-stromal cell interactions promote growth of pancreatic cancer. Cancer Res 2013;73:3927-37.

26 Ludwig T, Fisher P, Murty V, et al. Development of mammary adenocarcinomas by tissue-specific knockout of Brca2 in mice. Oncogene 2001;20:3937-48.

27 Guenterberg KD, Lesinski GB, Mundy-Bosse BL, et al. Enhanced anti-tumor activity of interferon-alpha in SOCS1-deficient mice is mediated by $\mathrm{CD}^{+}$and $\mathrm{CD} 8^{+} \mathrm{T}$ cells. Cancer Immunol Immunother 2011;60:1281-8.

28 Sartor MA, Tomlinson CR, Wesselkamper SC, et al. Intensity-based hierarchical Bayes method improves testing for differentially expressed genes in microarray experiments. BMC Bioinformatics 2006:7:538.

29 Gordon A, Glazko G, Qiu X, et al. Control of the Mean Number of False Discoveries, Bonferroni and Stability of Multiple Testing. Ann App/ Stat 2007;1:179-90

30 Slinker BK. The statistics of synergism. J Mol Cell Cardiol 1998;30:723-31.

31 Boj SF, Hwang $\mathrm{Cl}$, Baker LA, et al. Organoid models of human and mouse ductal pancreatic cancer. Cell 2015;160:324-38.

32 Collignon A, Perles-Barbacaru AT, Robert $S$, et al. A pancreatic tumor-specific biomarker characterized in humans and mice as an immunogenic onco-glycoprotein is efficient in dendritic cell vaccination. Oncotarget 2015;6:23462-79.

33 Pilon-Thomas S, Nelson N, Vohra N, et al. Murine pancreatic adenocarcinoma dampens SHIP-1 expression and alters MDSC homeostasis and function. PLoS One 2011;6:e27729.

34 Suzuki $K$, Ogura $M$, Abe $Y$, et al. Phase 1 study in Japan of siltuximab, an anti-IL-6 monoclonal antibody, in relapsed/refractory multiple myeloma. Int J Hematol 2015;101:286-94.

35 San-Miguel J, Bladé J, Shpilberg 0, et al. Phase 2 randomized study of bortezomib-melphalan-prednisone with or without siltuximab (anti-IL-6) in multiple myeloma. Blood 2014;123:4136-42.

36 Hunsucker SA, Magarotto V, Kuhn DJ, et al. Blockade of interleukin-6 signalling with siltuximab enhances melphalan cytotoxicity in preclinical models of multiple myeloma. Br J Haematol 2011:152:579-92.

37 Dijkgraaf EM, Santegoets SJ, Reyners AK, et al. A phase I trial combining carboplatin/doxorubicin with tocilizumab, an anti-IL-6R monoclonal antibody, and interferon- $\alpha 2 b$ in patients with recurrent epithelial ovarian cancer. Ann Oncol 2015;26:2141-9. 
38 Bayne LJ, Beatty GL, Jhala N, et al. Tumor-derived granulocyte-macrophage colony-stimulating factor regulates myeloid inflammation and $\mathrm{T}$ cell immunity in pancreatic cancer. Cancer Cell 2012;21:822-35.

39 Neesse A, Michl P, Frese KK, et al. Stromal biology and therapy in pancreatic cancer. Gut 2011;60:861-8.

40 Fearon DT. The carcinoma-associated fibroblast expressing fibroblast activation protein and escape from immune surveillance. Cancer Immunol Res 2014:2:187-93.

41 Feig C, Jones J0, Kraman M, et al. Targeting CXCL12 from FAP-expressing carcinoma-associated fibroblasts synergizes with anti-PD-L1 immunotherapy in pancreatic cancer. Proc Natl Acad Sci USA 2013;110:20212-17.

42 Lo A, Wang LC, Scholler J, et al. Tumor-Promoting Desmoplasia Is Disrupted by Depleting FAP-Expressing Stromal Cells. Cancer Res 2015;75:2800-10.

43 Rhim AD, Oberstein PE, Thomas DH, et al. Stromal Elements Act to Restrain, Rather Than Support, Pancreatic Ductal Adenocarcinoma. Cancer Cell 2014;25:735-47.
44 Lee JJ, Perera RM, Wang $\mathrm{H}$, et al. Stromal response to Hedgehog signaling restrains pancreatic cancer progression. Proc Natl Acad Sci USA 2014;111:E3091-100.

45 Özdemir BC, Pentcheva-Hoang T, Carstens JL, et al. Depletion of Carcinoma-Associated Fibroblasts and Fibrosis Induces Immunosuppression and Accelerates Pancreas Cancer with Reduced Survival. Cancer Cell 2014;25:719-34.

46 Dodge IL, Carr MW, Cernadas M, et al. IL-6 production by pulmonary dendritic cells impedes Th1 immune responses. J Immunol 2003;170:4457-64.

47 Wang $Y$, van Boxel-Dezaire AH, Cheon $\mathrm{H}$, et al. STAT3 activation in response to IL-6 is prolonged by the binding of IL-6 receptor to EGF receptor. Proc Natl Acad SCi USA 2013;110:16975-80.

48 Vassilakopoulou M, Avgeris M, Velcheti V, et al. Evaluation of PD-L1 Expression and Associated Tumor-Infiltrating Lymphocytes in Laryngeal Squamous Cell Carcinoma. Clin Cancer Res 2015:22:704-13.

49 Herbst RS, Soria JC, Kowanetz M, et al. Predictive correlates of response to the anti-PD-L1 antibody MPDL3280A in cancer patients. Nature 2014;515:563-7. 